\title{
Hippocampal-Dorsolateral Prefrontal Coupling as a Species-Conserved Cognitive Mechanism: A Human Translational Imaging Study
}

\author{
Florian Bähner ${ }^{1,2}$, Charmaine Demanuele ${ }^{1,2}$, Janina Schweiger ${ }^{2}$, Martin F Gerchen ${ }^{1,3}$, Vera Zamoscik ${ }^{3}$, \\ Kai Ueltzhöffer ${ }^{1,4}$, Tim Hahn ${ }^{4}$, Patric Meyer ${ }^{1,5}$, Herta Flor ${ }^{1,5}$, Daniel Durstewitz ${ }^{1,2}$, Heike Tost ${ }^{2}$, \\ Peter Kirsch ${ }^{1,3}$, Michael M Plichta ${ }^{2,6}$ and Andreas Meyer-Lindenberg*, 1,2,6 \\ 'Bernstein Center for Computational Neuroscience Heidelberg-Mannheim, Central Institute of Mental Health, Medical Faculty Mannheim, \\ Heidelberg University, Mannheim, Germany; ${ }^{2}$ Department of Psychiatry and Psychotherapy, Central Institute of Mental Health, Medical Faculty \\ Mannheim, Heidelberg University, Mannheim, Germany; ${ }^{3}$ Department of Clinical Psychology, Central Institute of Mental Health, Medical Faculty \\ Mannheim, Heidelberg University, Mannheim, Germany; ${ }^{4}$ Department of Psychology, Goethe University, Frankfurt am Main, Germany; ${ }^{5}$ Institute \\ of Cognitive and Clinical Neuroscience, Central Institute of Mental Health, Medical Faculty Mannheim, Heidelberg University, Mannheim, \\ Germany
}

\begin{abstract}
Hippocampal-prefrontal cortex (HC-PFC) interactions are implicated in working memory (WM) and altered in psychiatric conditions with cognitive impairment such as schizophrenia. While coupling between both structures is crucial for WM performance in rodents, evidence from human studies is conflicting and translation of findings is complicated by the use of differing paradigms across species. We therefore used functional magnetic resonance imaging together with a spatial WM paradigm adapted from rodent research to examine HC-PFC coupling in humans. A PFC-parietal network was functionally connected to hippocampus (HC) during task stages requiring high levels of executive control but not during a matched control condition. The magnitude of coupling in a network comprising HC, bilateral dorsolateral PFC (DLPFC), and right supramarginal gyrus explained one-fourth of the variability in an independent spatial WM task but was unrelated to visual WM performance. HC-DLPFC coupling may thus represent a systems-level mechanism specific to spatial WM that is conserved across species, suggesting its utility for modeling cognitive dysfunction in translational neuroscience.
\end{abstract} Neuropsychopharmacology (20I5) 40, I674-I68I; doi:I0.I038/npp.20I5.I3; published online 4 February 2015

\section{INTRODUCTION}

A translational neuroscience approach to drug discovery depends on valid animal models, which are difficult to define in psychiatric disorders with human-specific features. One approach to this conundrum is to identify species-conserved systems-level mechanisms implicated in disease (Meyer-Lindenberg, 2010). Electrophysiological recordings in rodents have established a role for hippocampal-prefrontal (HC-PFC) interactions during spatial working memory (WM) performance (Floresco et al, 1997; Gordon, 2011; Jones and Wilson, 2005). Specifically, theta coherence between both structures is elevated during increased WM demands (Jones and Wilson, 2005), and

* Correspondence: Dr A Meyer-Lindenberg, Department of Psychiatry and Psychotherapy, Central Institute of Mental Health, Medical Faculty Mannheim, University of Heidelberg, J5, Mannheim 68159, Germany, Tel: +49621 1703 200।, Fax: +4962। 1703 2005,

E-mail: A.Meyer-Lindenberg@zi-mannheim.de

${ }^{6}$ These authors contributed equally to this work.

Received | October 2014; revised | December 2014; accepted 22 December 2014; accepted article preview online 12 January 2015 animals show decreased coupling during error trials (Benchenane et al, 2010; Jones and Wilson, 2005). In the human imaging literature, however, the role of $\mathrm{HC}-\mathrm{PFC}$ coupling during WM is controversial, as studies have found both decreased and increased coupling of $\mathrm{HC}$ and lateral PFC during conditions with a high WM load (Axmacher et al, 2008; Finn et al, 2010; Rissman et al, 2008). Outside cognitive neuroscience, clarifying the role of HC-dorsolateral PFC (DLPFC) interactions is of clinical interest, as altered connectivity between those structures has been shown to occur in schizophrenia (Meyer-Lindenberg et al, 2005) and in subjects at risk for this illness (Esslinger et al, 2009).

The implications of findings derived from animal electrophysiology and human imaging are difficult to reconcile. More specifically, it has been proposed that uncoupling may be beneficial for human WM performance as it leads to an inhibition of interfering cognitive processes, such as parallel encoding of stimuli in episodic memory (Meyer-Lindenberg et al, 2005). However, this hypothesis is mainly based on results from the n-back task that does not specifically challenge spatial-relational WM and for which hippocampal 
activity may not be crucial (Esslinger et al, 2009). One of the sources of these trans-species discrepancies may thus be related to the specific WM tasks used. In rodents, one of the best studied spatial WM paradigms is the radial arm maze (RAM) task (Olton and Samuelson, 1976). Lesion studies show that both the HC, PFC, and an intact HC-PFC structural connectivity are required for successful performance in a delayed version of the RAM (Floresco et al, 1997; Seamans et al, 1995). Patients with selective hippocampal damage show impairments during performance of the RAM that are comparable to animals with HC lesions (Goodrich-Hunsaker and Hopkins, 2010). Although the effect of DLPFC lesions on RAM performance in humans is unknown, there is sound evidence that such lesions lead to impaired cognitive control (Gläscher et al, 2012) and deficient executive processes during WM (Barbey et al, 2013).

In an attempt to bridge the discrepant findings revealed by rodent and human research, we used a virtual reality version of the RAM task to study blood oxygen leveldependent (BOLD) HC-PFC functional connectivity and their relation to WM performance in humans. We hypothesized that under comparable task demands, significant HC-DLPFC coupling would occur and would be selective for task stages with a strong spatial WM component. Furthermore, we expected that task-induced HC-DLPFC connectivity values should predict spatial WM performance. Conversely, HC-DLPFC coupling in a task-free state (ie, during the resting state) should not be related to spatial WM performance. Finally, we predicted that if discrepant findings on HC-DLPFC coupling are indeed related to task properties/WM modality (stronger spatial-relational component in rodent as compared with human WM paradigms), the magnitude of coupling should predict spatial but not visual WM performance in independent tasks. Our findings indeed provide evidence for these hypotheses, and therefore for a species-conserved role of HC-DLPFC connectivity for spatial WM capacity.

\section{MATERIALS AND METHODS}

\section{Subjects}

A sample of 125 healthy right-handed German subjects without a history of mental disorder was recruited at two sites (Mannheim and Heidelberg). All subjects gave written informed consent and received an amount of $50 €$ for participation. A total of 28 subjects had to be excluded (five subjects did not complete all study parts, seven were excluded due to incidental anatomical findings or previously unreported somatic disorders, five due to image artifacts, and 11 subjects due to poor behavioral performance). The results are thus reported for 97 individuals (site: 34 subjects in Mannheim, 63 subjects in Heidelberg; gender: 46 women and $51 \mathrm{men}$; and age: $30.7 \pm 9.4$ years). The subjects first completed a neuropsychological assessment battery (CANTAB, Cambridge Cognition, Cambridge, $\mathrm{UK}$ ) and were then invited to participate in two MRI scans where anatomical and functional data (resting state scan and a task battery consisting of four paradigms) were acquired. The order of the tasks was balanced across subjects. The study was approved by the local ethics committees of the University of Heidelberg.

\section{Task}

A virtual reality version of a delayed win-shift RAM task (Floresco et al, 1997) was implemented for use in humans. The software was written in $\mathrm{C}^{++}$, using the 3D rendering engine OGRE (http://www.ogre3d.org/). During the acquisition of functional magnetic resonance imaging (fMRI) data, subjects used an fMRI-compatible four-button diamond fiber optic response pad (Current Designs, Philadelphia, $\mathrm{PA}$ ) to navigate through a virtual maze (consisting of 12 equally spaced arms radiating from a central platform) from the perspective of a ground-level observer. The maze was surrounded by various landmarks (telephone booth, church, tractor, tree, and houses). The paradigm was completely self-paced and consisted of three task (training, delay, and test) and matched control phases. Subjects encoded the position of hidden gold coins that they were asked to retrieve after a 30-s delay period. In order to increase homogeneity within and between subjects, we designed the task such that a ceiling effect could be observed with respect to performance in the test phase (median: 0 errors/test phase, $P_{25}: 0, P_{75}: 0, N=108$ subjects; see Figure 1, Supplementary Figure $S 1$ and Supplementary Materials and Methods for more details). We excluded subjects from data analysis if they committed more than four errors during a test phase (corresponding to the $2.5 \%$ worst performance values).

\section{Data Acquisition and Preprocessing of Task-Related Data}

BOLD fMRI data were acquired on two 3T Siemens Trio scanners (München, Germany) in Mannheim and Heidelberg using standard acquisition (gradient-echo echoplanar imaging (EPI)) and preprocessing procedures (Supplementary Materials and Methods).

\section{Task-Dependent Functional Connectivity}

A bilateral mask of the HC taken from the Harvard-Oxford probabilistic atlas (http://fsl.fmrib.ox.ac.uk/fsl/fslwiki/Atlases/) was thresholded at $80 \%$ and used as a seed for all connectivity analyses. A bilateral mask of the DLPFC (middle frontal gyrus from the Harvard-Oxford probabilistic atlas, thresholded at 50\%) was used to evaluate HC-DLPFC connectivity at the region of interest (ROI) level. Functional connectivity was estimated using the beta series correlation method (Rissman et al, 2004). Briefly, a general linear model (GLM) was built containing a unique regressor for every trial of each condition. Trial-specific beta values were then sorted by condition and concatenated to generate a beta time series. A beta series thus reflects single-trial variability of responses for a specific condition. Functional connectivity was computed by correlating the beta series of the seed (mean beta series of all seed voxels) with the beta series of all other voxels in the brain (Supplementary Materials and Methods).

\section{Control Analyses}

Several analyses were conducted to rule out that HC-DLPFC coupling is driven by the variable duration of individual 
a

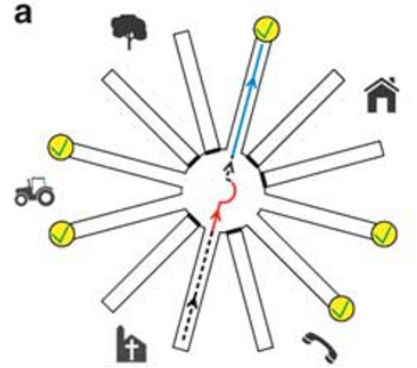

b

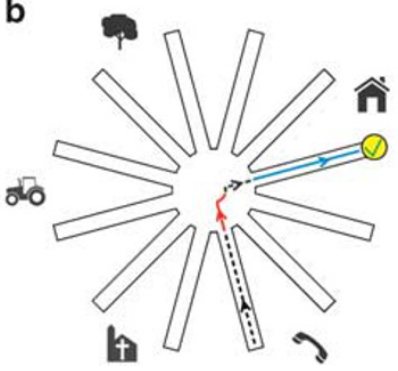

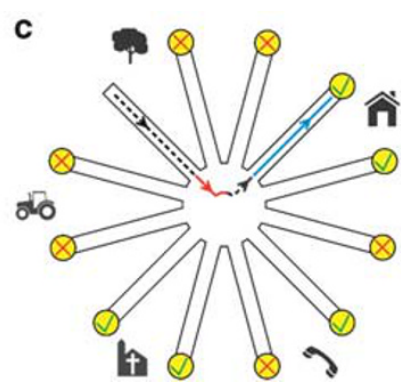

Figure I Task description and analysis strategy. Schematic drawing of the RAM during training, test, and the control phase. Note that subjects navigate the maze from the perspective of a ground-level observer (Supplementary Figure SI). (a) During training, subjects need to collect all accessible gold coins and encode the position of six STOP signs using landmarks that surround the maze. (b) In the test phase, subjects have to retrieve six hidden gold coins that are located at the end of the previously blocked arms. (c) In the visuomotor control, subjects have to collect gold coins with green check marks and avoid coins with a red cross. Both control/training and training/test are separated by a 30 s delay. All subjects $(N=97)$ completed the RAM task four times in a row and the set of baited arms changed each time. Typical trajectories of subjects in each task stage are shown. After collecting a gold coin, subjects leave the arm and navigate on the platform (red line) before entering another arm in order to retrieve the next gold coin (blue line). Spatial processing on the platform differs between task stages such that subjects either simply navigate (control) or additionally engage in spatial WM encoding (training)/retrieval (test). Functional connectivity was estimated using the beta series correlation method (Rissman et al, 2004) in which a GLM is built containing a unique regressor for every trial of each condition. Our model thus contained $24(6 \times 4)$ regressors for each of the six conditions shown in a-c. Functional connectivity is expressed as the correlation of the single-trial variability of responses for a specific condition in the $\mathrm{HC}$ and the rest of the brain.

trials or visuomotor effects rather than spatial WM processing (Supplementary Materials and Methods/Results, and Supplementary Figure S3). Site, age, and gender were also entered in the group-level analyses as covariates. There were no age or gender effects on HC connectivity both at the whole-brain level or within the probabilistic DLPFC ROI.

\section{Resting State Connectivity}

For the resting state scan, a 5:08 min EPI sequence was acquired in the same MRI session as the RAM data. The same HC mask was used as a seed for both task-dependent and task-free connectivity data (see Supplementary Materials and Methods for details).

\section{Statistical Inference}

Results that were significant either at the whole-brain level or within a probabilistic DLPFC ROI (middle frontal gyrus from the Harvard-Oxford probabilistic atlas, bilateral mask thresholded at 50\%) after correcting for multiple comparisons $(p<0.05$ family-wise error/FWE correction) are reported. Thresholded F- and t-maps were surface rendered using the SPM SurfRend toolbox (http://spmsurfrend.sourceforge.net). If data were normally distributed, descriptive statistics were given as mean \pm SD and parametric tests were used. Otherwise data were reported as median and first and third quartile $\left(P_{25}, P_{75}\right)$, and non-parametric tests were used. Outliers are defined as any data point $>1.5$ interquartile ranges below the first quartile or above the third quartile.

\section{Results}

\section{Behavioral Characterization of Spatial Processing Across Task Stages}

Debriefing after the scan confirmed that all subjects used a landmark-based encoding strategy that has been shown to depend on the HC in both rodents and humans
(Goodrich-Hunsaker and Hopkins, 2010; Iaria et al, 2003; Packard and McGaugh, 1996). We used several behavioral parameters to characterize spatial processing on the platform across task stages (Supplementary Table S1). These analyses confirmed that spatial WM processing during the experimental conditions (WM encoding/training and WM retrieval/test) was higher than that during the visuomotor control phase (training $>$ test $>$ control; two-tailed Wilcoxon-signed rank tests). Subjects, thus, not only used a spatial strategy to solve the task but the level of spatial WM processing was also clearly different between task stages.

\section{The Bilateral HC Couples to a Frontoparietal Control Network During Spatial WM Processing}

HC-PFC coupling in rodents has been reported to be higher during spatial processing on the platform of a maze as opposed to navigation and reward processing within an arm (Benchenane et al, 2010; Jones and Wilson, 2005). Inspired by these findings, we used a beta series correlation approach (Rissman et al, 2004) to contrast bilateral hippocampal connectivity while subjects where navigating on $v s$ off the platform during the training, test, and control phase (Figure 1 and Supplementary Materials and Methods). We first computed a $2 \times 3$ full factorial model with the factors location (on $v s$ off the platform) and task phase (training, test, and control) to test whether HCDLPFC coupling is elevated during spatial processing on the platform in a task stage-dependent manner. Indeed, the location $\times$ task phase interaction was significant within the DLPFC (Figure 2a; F-test, $p<0.05$ ROI-FWE correction; peak voxel Montreal Neurological Institute (MNI) coordinates in left DLPFC $-45,14,48$ and $-51,20,33)$. Post hoc $t$-tests using the mean value of all significant DLPFC voxels showed that HC-DLPFC connectivity on $v s$ off the platform was stronger in training as compared with test and control but not different between the latter (Figure 2b; training: $1.0 \pm 1.5$, test: $0.14 \pm 1.3$, and control: $0.0 \pm 1.4$; training $v s$ test: $p=3.5 \times 10^{-5}$, training $v s$ control: $p=7.1 \times 10^{-6}$, test 
a

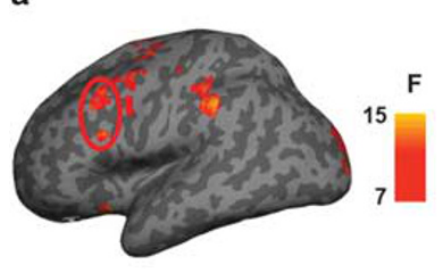

\section{b}
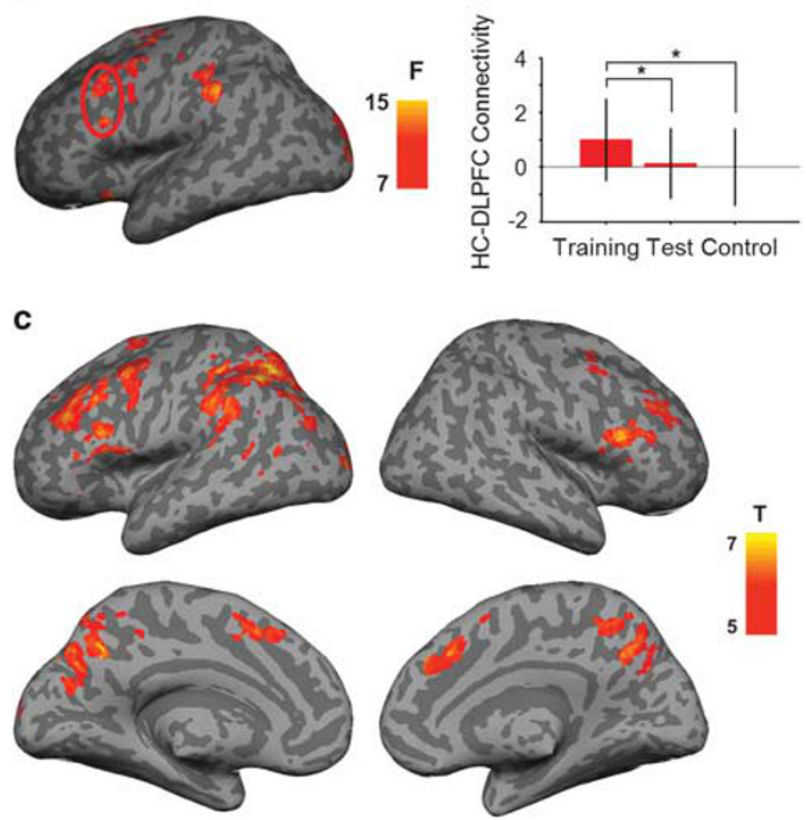

Figure 2 Bilateral HC-DLPFC connectivity is strongest during spatial WM encoding. (a) The location (on vs off the platform) $\times$ task phase (training, test, and control) interaction was used to test whether HCDLPFC connectivity is selective for spatial WM processing on the platform. The F-test used to evaluate this interaction was significant within the DLPFC mask (ROI-FWE correction; results are displayed at the wholebrain level with $p<0.001$ uncorrected, $k=50$; the left DLPFC is highlighted). (b) Post hoc t-tests using the mean connectivity of the significant DLPFC interaction cluster described above. Functional connectivity is contrasted on vs off the platform across task stages. HC-DLPFC coupling is strongest during spatial WM encoding (connectivity values given as mean $\pm S D$, asterisks indicate $p<0.00 \mathrm{I}$ ). Note that at the behavioral level, subjects also show the highest level of spatial processing in training (Supplementary Table SI). (c) Functional connectivity of the $\mathrm{HC}$ with the rest of the brain is contrasted on vs off the platform in training. Note positive $\mathrm{HC}$ coupling with a distributed prefrontal-parietal cognitive control network during WM encoding. Results are shown at $p<0.05$ whole-brain FWE corrected, $k=20$.

$v s$ control: $p=0.49$; two-tailed paired $t$-tests). This effect also persisted when taking the mean of the entire DLPFC ROI (training: $0.8 \pm 1.4$, test: $0.3 \pm 1.1$, and control: $0.2 \pm 1.3$; training $v s$ test: $p=0.004$, training $v s$ control: $p=0.002$, test vs control: $p=0.41$ ).

At the whole-brain level, the bilateral HC was positively coupled to a network comprising bilateral PFC (including both DLPFC and ventrolateral PFC/VLPFC), left superior parietal lobule, cingulate gyrus/supplementary motor area, precuneus, left middle temporal gyrus, as well as primary visual cortex in training $(p<0.05$ FWE correction; Figure $2 \mathrm{c}$ and Supplementary Table S2). During the test phase, hippocampal connectivity was found mainly in the left hemisphere within inferior parietal lobule, frontal eye fields, insula, and DLPFC and also in the right VLPFC (Supplementary Figure S2 and Supplementary Table S3). The frontal eye fields are part of an attention circuit (Power et al, 2011) implicated in spatial WM, which has been suggested to act 'as a focus of attention that cycles through spatial locations held in mind' (p.973 in Nee and Jonides, 2009).
Interaction of the $\mathrm{HC}$ with the frontal eye fields may thus be beneficial for the sequential retrieval of hidden gold coins during the test phase. No hippocampal connectivity was observed during the matched control task with similar visuomotor demands but without a relevant WM component. Additional control analyses confirmed that the reported connectivity findings are driven by different levels of spatial WM processing and cannot be explained by either visuomotor effects or increased behavioral variance owing to our task design (Supplementary Results and Supplementary Figure S3). Hippocampal coupling thus occurs only during WM-related task stages and is strongest with networks related to executive control (DLPFC, inferior parietal lobule/intraparietal sulcus, precuneus, anterior cingulate, and insula) and spatial attention (dorsal attention network: frontal eye fields and superior parietal lobule/intraparietal sulcus; Cole and Schneider, 2007; Power et al, 2011).

\section{Functional Connectivity of a Network Comprising HC, Bilateral DLPFC and Right Inferior Parietal Lobule During Training Predicts Spatial but not Visual WM Performance}

In the rodent literature, evidence has accumulated that $\mathrm{HC}-\mathrm{PFC}$ coupling is related to spatial WM performance (Gordon, 2011; Jones and Wilson, 2005; Sigurdsson et al, 2010). Owing to the ceiling effect in our paradigm (with respect to performance in the test phase; Supplementary Materials and Methods), a direct correlation of connectivity and RAM performance was not possible. Therefore, we selected two different measures of WM performance from a neuropsychological assessment all subjects underwent outside the scanner to test for a potential relationship with HCPFC coupling. More specifically, values for spatial (spatial span) and visual (percentage of correct responses in a delayed matching-to-sample paradigm) WM performance were used as regressors in the group-level model examining hippocampal functional connectivity during the spatial WM encoding phase of the RAM. This analysis yielded a spatially confined network with peak voxels in bilateral DLPFC (peak voxel MNI coordinates: left DLPFC - 36, 41, 39 and right DLPFC 24, 56, 36) and the right supramarginal gyrus $(48,-28,27)$ that showed a strong (significant after FWE whole-brain correction) positive correlation of hippocampal connectivity with spatial WM capacity. In other words, higher connectivity values in these clusters went along with better spatial WM performance outside the scanner (red network in Figure 3a). The coupling strength of this network explained $25.4 \%$ of the variation in spatial WM performance in the independent task (Pearson's $r=0.504$; Figure $3 \mathrm{~b}$ ). In contrast, this network was uncorrelated with visual WM performance (Pearson's $r=-0.030$; Supplementary Figure S4). No voxel-wise positive or negative correlation was found for $\mathrm{HC}$ connectivity with visual WM performance. Control analyses confirmed that these findings cannot be explained by visuomotor effects (Supplementary Results). In order to compare the relationship between HC-DLPFC connectivity and spatial vs visual WM in an unbiased fashion, we also computed the correlation between HC-DLPFC connectivity (mean of HC connectivity within the probabilistic DLPFC ROI) and spatial (Pearson's $r=0.286$, 
a
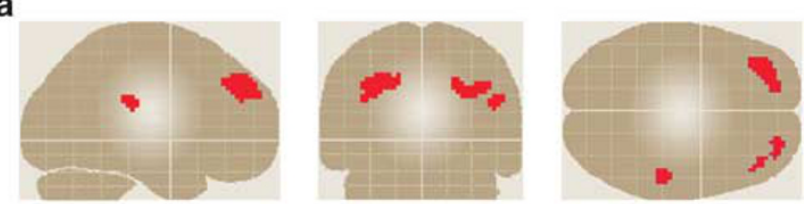

b

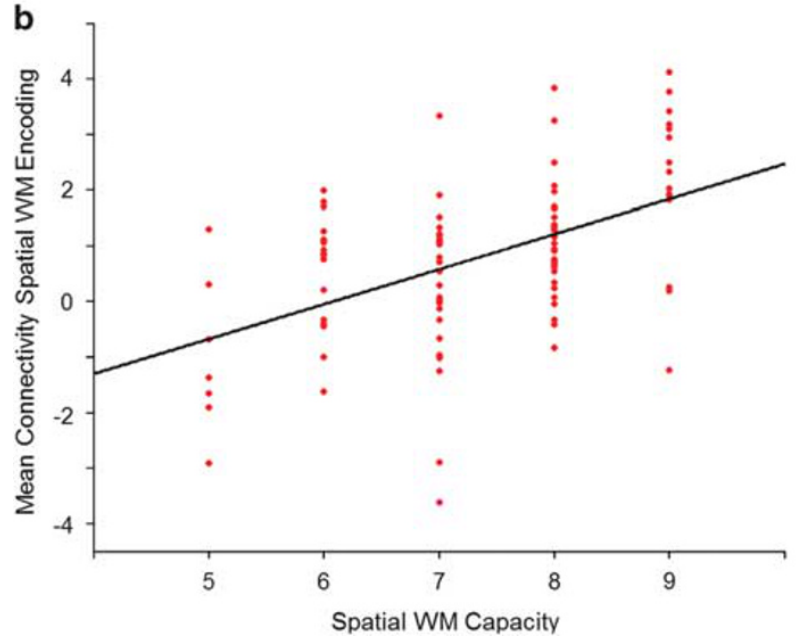

Figure $3 \mathrm{HC}-\mathrm{DLPFC}$ connectivity during training predicts spatial but not visual WM performance. (a) Behavioral performance in a spatial (spatial span) and a visual WM task (delayed matching-to-sample) measured outside the scanner was entered as additional regressors in the group-level connectivity model. The correlation of spatial WM capacity and HC functional connectivity during training is highest in a network comprising bilateral DLPFC and right supramarginal gyrus (red network; significant at the whole-brain level after FWE correction but displayed at $p<10^{-4}$ uncorrected, $k=20$ ). No voxel-wise positive or negative correlation was found for $\mathrm{HC}$ connectivity with visual WM performance. (b) Scatter plot showing the correlation of the HC-DLPFC-parietal connectivity in training with spatial WM performance. Each dot represents the mean connectivity value of the red network shown in panel a and the respective spatial WM capacity for a single subject. A linear fit is used to illustrate the strength of the correlation. Note that the magnitude of HC-DLPFCparietal coupling explains $\sim$ I/4 of the variation in spatial WM capacity (Pearson's $r=0.504$ ).

$p=0.005$ ) or visual WM (Pearson's $r=-0.054, p=0.60$ ). A direct comparison of both correlation coefficients confirmed the specific association of hippocampal connectivity with spatial WM (two-tailed test for the difference between two dependent correlations: $Z$-score $=2.61, \quad p=0.009$; Steiger, 1980).

Previous work indicated that task-induced activation and connectivity patterns may be equally expressed in the resting state (Cole et al, 2014; Smith et al, 2009). We thus examined whether spatial WM performance outside the scanner could also be predicted by HC-DLPFC coupling in a task-free state. Resting state scans were obtained from all individuals during the same session and a seed-based approach was used to evaluate hippocampal connectivity (using the same seed as for task-dependent connectivity). This analysis showed that there was no significant correlation of either spatial or visual WM performance with hippocampal connectivity during the resting state (both at the whole-brain level and within the DLPFC ROI).

\section{Discussion}

This study used a self-paced translational WM paradigm together with a functional connectivity analysis inspired by the rodent literature to study the role of HC-DLPFC coupling in spatial WM in humans. We compared HC-DLPFC connectivity across task stages and found that it was strongest during spatial WM encoding, the phase during which we observed the highest level of spatial processing at the behavioral level. The HC also coupled to other nodes of a frontoparietal control network during both encoding and retrieval, but no connectivity was observed during a matched control condition that lacked a WM component. Several analyses that controlled for intra- and interindividual variation of non-WM-related task aspects showed that HC-DLPFC connectivity cannot be explained by increased behavioral variance related to the task design or by visuomotor effects. Furthermore, the magnitude of functional coupling of a network comprising HC, bilateral DLPFC, and right supramarginal gyrus during training explained roughly one-fourth of the variability in spatial WM capacity recorded outside the scanner but was unrelated to visual WM accuracy. This effect was specific for task-induced connectivity because the magnitude of HC-DLPFC coupling in the resting state did not predict spatial WM capacity. This suggests that the interaction of frontoparietal control networks with hippocampal signals may be important for encoding of goal-directed spatial behaviors.

\section{HC-PFC Coupling During WM-Good or Bad?}

Previous work in rodents and humans has yielded conflicting results regarding the role of $\mathrm{HC}-\mathrm{PFC}$ coupling in WM. Research in rodents has shown increasing evidence that HC-PFC coupling is required for spatial WM performance (Gordon, 2011; Jones and Wilson, 2005; Sigurdsson et al, 2010). Findings in humans, however, draw a different picture: several studies have shown that HC-DLPFC coupling seems to decrease with increasing WM load (Axmacher et al, 2008; Esslinger et al, 2009). In a longitudinal study, HC-DLPFC connectivity generally decreased across adolescence but persisted in adults during visual WM with high task demands (Finn et al, 2010). Impaired WM is a core cognitive deficit in schizophrenia (Barch and Ceaser, 2012), and both schizophrenic patients, their unaffected siblings, and healthy risk gene carriers have been shown to display persistent HC-DLPFC coupling during high as compared with low WM load (Esslinger et al, 2009; Meyer-Lindenberg et al, 2005; Rasetti et al, 2011), thereby making it one of the best validated endophenotypes for schizophrenia to date. Based on these findings, it has been proposed that uncoupling rather than coupling of $\mathrm{HC}$ and DLPFC is a signature of successful WM performance (Meyer-Lindenberg et al, 2005). While recruitment of both HC and DLPFC has been reported for imaging studies that examined maze-based navigation (Astur et al, 2005; Iaria et al, 2003; Iglói et al, 2010; Marsh et al, 2010), the role of HC-PFC interactions during maze performance has not been tested previously.

Our findings now suggest that these discrepancies are primarily related to WM modality if translational neuroimaging is used to control for task differences across species. While rodent tasks use spatial-relational challenges, 
non-spatial stimulus material is used in the majority of human WM studies. Using a virtual reality version of a rodent task, we found that the magnitude of coupling between HC, DLPFC, and right supramarginal gyrus significantly predicted spatial WM capacity in an independent task indicating that this type of coupling also has a role in humans. The supramarginal gyrus has been related to both spatial attention and WM (Schenkluhn et al, 2008; Silk et al, 2010), as well as planning of goal-directed actions (Tunik et al, 2008). Findings from a recent study using a HC-dependent memory paradigm in humans indicate that volitional control as opposed to passive learning results in better spatial-relational memory and is linked to a functionally connected network comprising HC and DLPFC (Voss et al, 2011). The authors argue that brain regions involved in cognitive control may need to interact with the memory system to optimize learning and goal-directed behavior. This could be because both systems operate within a limited bandwidth and therefore need to determine which information will be attended (Voss et al, 2011). We propose that similar processes may also be involved in spatial WM (Supplementary Discussion).

\section{Limitations}

Although our findings thus suggest a species-conserved mechanism specifically for spatial WM, several caveats remain. First, the relationship between the electrophysiological readouts commonly used in rodents and corresponding functional MRI measures are complicated and understudied (Logothetis, 2008). Furthermore, rodent electrophysiology studies assess connectivity changes at a much finer timescale than what can be done using fMRI methods (where the signal is typically averaged over an entire trial). Few studies investigated the neural correlates of BOLD connectivity or co-activation of brain regions. One study in monkeys directly examined the neural basis of interareal BOLD coupling in visual areas. They found that mainly low-frequency neural oscillations contributed to BOLD-positive functional connectivity (Wang et al, 2012). Another group used hippocampal depth electrode recordings together with fMRI measurements in non-human primates and found that cortical areas are activated by certain hippocampal oscillations (Logothetis et al, 2012). In addition, our data do show WM-related positive HC-DLPFC coupling in a translational task for which the interaction of $\mathrm{HC}$ and PFC is necessary in rodents (Floresco et al, 1997).

Second, identifying homologies in the interacting brain regions across species is nontrivial. Although this is certainly a controversial topic (Uylings et al, 2003), many studies have now shown that WM functions that are mediated by the lateral PFC in primates involve the medial PFC in rodents (Euston et al, 2012). HC-medial PFC connectivity during WM in rodents may therefore translate into coupling of the $\mathrm{HC}$ to ventral and dorsal aspects of the lateral PFC in humans. Although most data examining HC connectivity during WM have found an uncoupling of the DLPFC, there is some evidence that coupling of the HC to the VLPFC may be related to the maintenance aspect of WM in humans (Cohen, 2011; Poch et al, 2011; Rissman et al, 2008; but see: Axmacher et al, 2008). Positive coupling of HC and VLPFC was also detected during spatial WM processing in the RAM task. However, only HC-DLPFC coupling during WM encoding was related to spatial WM capacity.

Finally, it should be noted that HC-PFC theta coherence in rodents has mainly been reported for the test and not the training phase of spatial WM tasks (Jones and Wilson, 2005; Sigurdsson et al, 2010). These studies used (delayed) spatial alternation tasks in a T-maze consisting of a sample phase in which animals were directed to enter one of two goal arms (no WM component) and a test phase in which animals had to remember the arm visited during the sample phase and enter the opposite arm. Such paradigms thus do not contain a training phase with a strong WM component. Disconnection lesions have shown that HC-PFC structural connectivity is required for performance in the retrieval phase of the delayed win-shift RAM (the paradigm we used in this study). However, to the best of our knowledge, the effect of such lesions has not been tested for the encoding phase of the RAM. Measurements of HC-PFC theta coherence during both the training and test phase of the RAM in rodents as well as the evaluation of HC-PFC BOLD connectivity during maze-based WM tasks with a predominant retrieval component in humans could help to clarify this issue.

\section{Summary and Outlook}

In summary, this study used a translational approach to show that the HC positively couples to a frontoparietal control network during spatial WM processing. Coupling strength of this network during training significantly predicted spatial WM capacity in an independent task but showed no association with visual WM performance. This indicates that HC-DLPFC coupling may be a systems-level correlate specific to spatial WM performance that can be found in several species. Impaired information processing during WM has been found to be a core cognitive deficit in schizophrenia (Barch and Ceaser, 2012) and has also been observed during normal aging (Nagel et al, 2009). Virtual navigation paradigms have already been successfully used in aged subjects and in clinical populations (Konishi et al, 2013; Sneider et al, 2013; Spieker et al, 2012; Yuan et al, 2014). Although there are currently no established treatments for such deficits, the use of this translational paradigm in affected populations is expected to help identify the neural correlates of WM impairment and may ultimately inform novel treatments (Millan et al, 2012). It is important to keep in mind that this paradigm may have to be modified for different populations depending on the exact research question being asked. The ceiling effect (with respect to test performance) in this study was introduced to increase the homogeneity of our data set, which was desirable for the imaging analyses. However, the level of task difficulty may have to be adjusted for a specific population if translational research aims to either use performance during retrieval as a measure of treatment effects on the spatial WM system or if this parameter is used to compare spatial WM performance across populations. Similarly, the use of HC-DLPFC coupling during training as a biomarker for treatment effects on spatial WM networks can only be interpreted in conjunction with external behavioral parameters if there is no effect on maze performance. 


\section{FUNDING AND DISCLOSURE}

This work was funded through a grant from the German Ministry of Education and Research (BMBF, 01GQ1003B) and from the German Science Foundation (DFG, Du 354/61 , Du 354/7-2). The funders had no role in study design, data collection and analysis, decision to publish, or preparation of the manuscript. AM-L has received consultant fees and travel expenses from Alexza Pharmaceuticals, AstraZeneca, Bristol-Myers Squibb, Defined Health, Decision Resources, Desitin Arzneimittel, Elsevier, F. Hoffmann-La Roche, Gerson Lehrman Group, Grupo Ferrer, Les Laboratoires Servier, Lilly Deutschland, Lundbeck Foundation, Outcome Sciences, Outcome Europe, PriceSpective, and Roche Pharma, and has received speaker's fees from Abbott, AstraZeneca, BASF, Bristol-Myers Squibb, GlaxoSmithKline, Janssen- Cilag, Lundbeck, Pfizer Pharma, and Servier Deutschland. The remaining authors declare no conflict of interest.

\section{ACKNOWLEDGEMENTS}

The task software was developed in collaboration with the Experimental Radiation Oncology group (Julia Ziegler and Professor Hesser, Mannheim). We thank Axel Schäfer for many helpful discussions.

\section{REFERENCES}

Astur RS, St Germain SA, Baker EK, Calhoun V, Pearlson GD, Constable RT (2005). fMRI hippocampal activity during a virtual radial arm maze. Appl Psychophysiol Biofeedback 30: 307-317.

Axmacher N, Schmitz DP, Wagner T, Elger CE, Fell J (2008). Interactions between medial temporal lobe, prefrontal cortex, and inferior temporal regions during visual working memory: a combined intracranial EEG and functional magnetic resonance imaging study. J Neurosci 28: 7304-7312.

Barbey AK, Koenigs M, Grafman J (2013). Dorsolateral prefrontal contributions to human working memory. Cortex 49: 1195-1205.

Barch DM, Ceaser A (2012). Cognition in schizophrenia: core psychological and neural mechanisms. Trends Cogn Sci 16: 27-34.

Benchenane K, Peyrache A, Khamassi M, Tierney PL, Gioanni Y, Battaglia FP et al (2010). Coherent theta oscillations and reorganization of spike timing in the hippocampal- prefrontal network upon learning. Neuron 66: 921-936.

Cohen MX (2011). Hippocampal-prefrontal connectivity predicts midfrontal oscillations and long-term memory performance. Curr Biol 21: 1900-1905.

Cole MW, Bassett DS, Power JD, Braver TS, Petersen SE (2014). Intrinsic and task-evoked network architectures of the human brain. Neuron 83: 238-251.

Cole MW, Schneider W (2007). The cognitive control network: integrated cortical regions with dissociable functions. Neuroimage 37: 343-360.

Esslinger C, Walter H, Kirsch P, Erk S, Schnell K, Arnold C et al (2009). Neural mechanisms of a genome-wide supported psychosis variant. Science 324: 605.

Euston DR, Gruber AJ, McNaughton BL (2012). The role of medial prefrontal cortex in memory and decision making. Neuron 76: 1057-1070.

Finn AS, Sheridan MA, Kam CLH, Hinshaw S, D’Esposito M (2010). Longitudinal evidence for functional specialization of the neural circuit supporting working memory in the human brain. J Neurosci 30: 11062-11067.
Floresco SB, Seamans JK, Phillips AG (1997). Selective roles for hippocampal, prefrontal cortical, and ventral striatal circuits in radial-arm maze tasks with or without a delay. J Neurosci 17: 1880-1890.

Gläscher J, Adolphs R, Damasio H, Bechara A, Rudrauf D, Calamia $M$ et al (2012). Lesion mapping of cognitive control and valuebased decision making in the prefrontal cortex. Proc Natl Acad Sci USA 109: 14681-14686.

Goodrich-Hunsaker NJ, Hopkins RO (2010). Spatial memory deficits in a virtual radial arm maze in amnesic participants with hippocampal damage. Behav Neurosci 124: 405-413.

Gordon JA (2011). Oscillations and hippocampal-prefrontal synchrony. Curr Opin Neurobiol 21: 486-491.

Iaria G, Petrides M, Dagher A, Pike B, Bohbot VD (2003). Cognitive strategies dependent on the hippocampus and caudate nucleus in human navigation: variability and change with practice. J Neurosci 23: 5945-5952.

Iglói K, Doeller CF, Berthoz A, Rondi-Reig L, Burgess N (2010). Lateralized human hippocampal activity predicts navigation based on sequence or place memory. Proc Natl Acad Sci USA 107: 14466-14471.

Jones MW, Wilson MA (2005). Theta rhythms coordinate hippocampal-prefrontal interactions in a spatial memory task. PLoS Biol 3: e402.

Konishi K, Etchamendy N, Roy S, Marighetto A, Rajah N, Bohbot VD (2013). Decreased functional magnetic resonance imaging activity in the hippocampus in favor of the caudate nucleus in older adults tested in a virtual navigation task. Hippocampus 23: 1005-1014.

Logothetis NK (2008). What we can do and what we cannot do with fMRI. Nature 453: 869-878.

Logothetis NK, Eschenko O, Murayama Y, Augath M, Steudel T, Evrard HC et al (2012). Hippocampal-cortical interaction during periods of subcortical silence. Nature 491: 547-553.

Marsh R, Hao X, Xu D, Wang Z, Duan Y, Liu J et al (2010). A virtual reality-based FMRI study of reward-based spatial learning. Neuropsychologia 48: 2912-2921.

Meyer-Lindenberg A (2010). From maps to mechanisms through neuroimaging of schizophrenia. Nature 468: 194-202.

Meyer-Lindenberg A, Olsen RK, Kohn PD, Brown T, Egan MF, Weinberger DR et al (2005). Regionally specific disturbance of dorsolateral prefrontal-hippocampal functional connectivity in schizophrenia. Arch Gen Psychiatry 62: 379-386.

Millan MJ, Agid Y, Brüne M, Bullmore ET, Carter CS, Clayton NS et al (2012). Cognitive dysfunction in psychiatric disorders: characteristics, causes and the quest for improved therapy. Nat Rev Drug Discov 11: 141-168.

Nagel IE, Preuschhof C, Li S-C, Nyberg L, Bäckman L, Lindenberger U et al (2009). Performance level modulates adult age differences in brain activation during spatial working memory. Proc Natl Acad Sci USA 106: 22552-22557.

Nee DE, Jonides J (2009). Common and distinct neural correlates of perceptual and memorial selection. Neuroimage 45: 963-975.

Olton DS, Samuelson RJ (1976). Remembrance of places passed: Spatial memory in rats. J Exp Psychol Anim Behav Process 2: 97-116.

Packard MG, McGaugh JL (1996). Inactivation of hippocampus or caudate nucleus with lidocaine differentially affects expression of place and response learning. Neurobiol Learn Mem 65: 65-72.

Poch C, Fuentemilla L, Barnes GR, Düzel E (2011). Hippocampal theta-phase modulation of replay correlates with configural-relational short-term memory performance. J Neurosci 31: 7038-7042.

Power JD, Cohen AL, Nelson SM, Wig GS, Barnes KA, Church JA et al (2011). Functional network organization of the human brain. Neuron 72: 665-678.

Rasetti R, Sambataro F, Chen Q, Callicott JH, Mattay VS, Weinberger DR (2011). Altered cortical network dynamics: a potential intermediate phenotype for schizophrenia and association with ZNF804A. Arch Gen Psychiatry 68: 1207-1217. 
Rissman J, Gazzaley A, D’Esposito M (2004). Measuring functional connectivity during distinct stages of a cognitive task. Neuroimage 23: 752-763.

Rissman J, Gazzaley A, D'Esposito M (2008). Dynamic adjustments in prefrontal, hippocampal, and inferior temporal interactions with increasing visual working memory load. Cereb Cortex 18: 1618-1629.

Schenkluhn B, Ruff CC, Heinen K, Chambers CD (2008). Parietal stimulation decouples spatial and feature-based attention. J Neurosci 28: 11106-11110.

Seamans JK, Floresco SB, Phillips AG (1995). Functional differences between the prelimbic and anterior cingulate regions of the rat prefrontal cortex. Behav Neurosci 109: 1063-1073.

Sigurdsson T, Stark KL, Karayiorgou M, Gogos JA, Gordon JA (2010). Impaired hippocampal-prefrontal synchrony in a genetic mouse model of schizophrenia. Nature 464: 763-767.

Silk TJ, Bellgrove MA, Wrafter P, Mattingley JB, Cunnington R (2010). Spatial working memory and spatial attention rely on common neural processes in the intraparietal sulcus. Neuroimage 53: 718-724.

Smith SM, Fox PT, Miller KL, Glahn DC, Fox PM, Mackay CE et al (2009). Correspondence of the brain's functional architecture during activation and rest. Proc Natl Acad Sci USA 106: 13040-13045.

Sneider JT, Gruber SA, Rogowska J, Silveri MM, Yurgelun-Todd DA (2013). A preliminary study of functional brain activation among marijuana users during performance of a virtual water maze task. J Addict 2013: 461029.

Spieker EA, Astur RS, West JT, Griego JA, Rowland LM (2012). Spatial memory deficits in a virtual reality eight-arm radial maze in schizophrenia. Schizophr Res 135: 84-89.

Steiger JH (1980). Tests for comparing elements of a correlation matrix. Psychol Bull 87: 245-251.

Tunik E, Lo O-Y, Adamovich SV (2008). Transcranial magnetic stimulation to the frontal operculum and supramarginal gyrus disrupts planning of outcome-based hand-object interactions. J Neurosci 28: 14422-14427.

Uylings HBM, Groenewegen HJ, Kolb B (2003). Do rats have a prefrontal cortex? Behav Brain Res 146: 3-17.

Voss JL, Gonsalves BD, Federmeier KD, Tranel D, Cohen NJ (2011). Hippocampal brain-network coordination during volitional exploratory behavior enhances learning. Nat Neurosci 14: $115-120$.

Wang L, Saalmann YB, Pinsk MA, Arcaro MJ, Kastner S (2012). Electrophysiological low-frequency coherence and cross-frequency coupling contribute to BOLD connectivity. Neuron 76: 1010-1020.

Yuan P, Daugherty AM, Raz N (2014). Turning bias in virtual spatial navigation: age-related differences and neuroanatomical correlates. Biol Psychol 96: 8-19.

Supplementary Information accompanies the paper on the Neuropsychopharmacology website (http://www.nature.com/npp) 